\title{
EL RETORNO DE LAS CARRERAS: UN ESTUDIO DE CASO DE LOS FACTORES QUE INCIDEN EN LAS REMUNERACIONES DE UNIVERSITARIOS RECIÉN TITULADOS ${ }^{1}$
}

\section{Roberto Schurch Santana ${ }^{2}$}

\section{RESUMEN}

El propósito del trabajo es ampliar la evidencia respecto de los factores que inciden en las remuneraciones de universitarios recién incorporados al mercado del trabajo, a partir de los salarios percibidos por titulados de una universidad privada no tradicional en un conjunto amplio de carreras en distintas áreas del conocimiento.

Usando modelos multinivel, los principales resultados indican el efecto de variables propias de las carreras afectando las remuneraciones de los titulados. Así, se aprecia una penalización salarial a las carreras feminizadas -que opera sobre hombres y mujeres-, un efecto positivo de la selectividad -más allá del rendimiento universitario observado- y un premio salarial a las carreras largas -más allá del retraso del estudiante en titularse-.

Palabras clave: Modelos multinivel, retorno salarial, duración de las carreras, feminización, selectividad.

\section{THE RETURN OF CAREERS: A CASE STUDY ABOUT FACTORS AFFECTING THE INCOME OF NEW UNIVERSITY GRADUATED STUDENTS}

\begin{abstract}
The aim of this paper is enhancing current evidence about factors that impact the income of university graduates who have joined the labor market recently. For that purpose, it uses data about incomes obtained by graduates from a non traditional private university, considering a wide range of degrees in different areas.

Using multilevel models, main findings show the effect of variables related to disciplines themselves affecting the income of graduates who studied those disciplines. In this sense, it is possible to see a wage punishment for graduates of female dominated disciplines- which is observable in the case of both women and men-, a positive effect of selectivity, beyond academic records of graduates, and also a wage recognition to programs that take more time to be completed, although students get their degree much later.
\end{abstract}

Keywords: Multilevel models, wage return, duration of bachelor's degrees, feminization, selectivity

1 Estudio desarrollado y enviado desde la Dirección de Análisis Institucional de la Universidad Diego Portales (UDP), no obstante el autor actualmente se desempeña en otro organismo. Así, se solicita dar crédito por la ejecución del trabajo a la institución originalmente indicada (UDP).

2 Sociólogo y Magíster en Sociología, Pontificia Universidad Católica de Chile, Santiago, Chile. Dirección de Análisis Institucional, Universidad Diego Portales. Contacto: rfschurc@gmail. com 


\section{EL RETORNO DE LAS CARRERAS: UN ESTUDIO DE CASO DE LOS FACTORES QUE INCIDEN EN LAS REMUNERACIONES DE UNIVERSITARIOS RECIÉN TITULADOS}

\section{Introducción}

En 2012, más de un millón de alumnos se encontraba matriculado en instituciones de educación superior en Chile. De ellos, más de la mitad estudiaba un programa en alguna de las 60 universidades existentes en el país (SIES, 2012a). El panorama actual contrasta radicalmente con lo observado previo a 1983, donde solo ocho universidades acogían a poco más de cien mil estudiantes (Meller y Quiroga, 2010a).

La masificación de la educación terciaria tiene como uno de los principales antecedentes la reforma a la educación superior llevada a cabo en 1980 que dio lugar a nuevas instituciones, a partir de la creación de Centros de Formación Técnica (CFT), Institutos Profesionales (IP), la descentralización de las universidades estatales y la aparición de universidades privadas sin Aporte Fiscal Directo (AFD).

Desde su implementación por ley, estas nuevas universidades privadas aumentaron progresivamente su participación en el sistema, pasando de 2.700 alumnos en 1983 a 374.000 en 2012, superando en número al total de matriculados en universidades del Consejo de Rectores (CRUCH) (SIES, 2012a; SIES, 2012b).

La nueva configuración del sistema en las últimas tres décadas evidencia el tránsito desde una coordinación estatal hacia una de mercado. Esta se caracteriza por la autorregulación, basada en las capacidades de los agentes descentralizados de actuar a partir de su comportamiento competitivo (Brunner y Uribe, 2007). En un arreglo de este tipo, el Estado tiende a mantener distancia, resguardando 
un número limitado de variables críticas, dentro de las cuales se encuentra el grado de información con que deben contar los agentes para la toma de decisiones, evitando en lo posible asimetrías entre estudiantes, apoderados, académicos, administradores de las instituciones y policymaker (encargados de diseñar políticas públicas).

En este contexto se han desarrollado algunas iniciativas que buscan responder a las demandas de información por parte de los agentes, intentando traspasar el círculo de la mera divulgación académica. Entre ellas se cuenta la plataforma Mi Futuro del Ministerio de Educación, la cual dispone de antecedentes acerca del financiamiento, orientación vocacional, oferta de instituciones y carreras, la empleabilidad e ingresos asociados a ellas. Esto último -cuál es la demora promedio en encontrar un trabajo y cuánto se percibe como salario- ha cobrado cada vez mayor relevancia, dada la presión por conocer indicadores de desempeño por sobre los recursos disponibles (dotación de profesiones, cantidad de libros, infraestructura académica, etc.) (Rappoport, Benavente y Meller, 2004). Esta presión se ha generado especialmente a favor de quienes demandan servicios educacionales, debido a que hoy en día el costo educacional recae principalmente en el estudiante y su grupo familiar, quienes deben contar con la mayor cantidad de antecedentes posibles respecto de la inversión que realizarán (Brunner, 2004).

Las investigaciones en la materia dan cuenta de retornos crecientes de la educación terciaria, especialmente para el caso de estudios superiores que concluyen con un título universitario (Mizala y Romaguera, 2004; Sapelli, 2009). La misma plataforma señalada (Mi futuro) indica en su sitio web que estudiantes universitarios ganan de tres a cinco veces más que los egresados de la Enseñanza Media, lo cual supera el retorno de los IP (dos veces) y CFT (50\% adicional a aquellos que solo terminan el colegio).

A su vez, la información disponible respecto de carreras y universidades ha proliferado en los últimos años, evidenciando una gran heterogeneidad en términos de selectividad, duración, exigencia, 
grado de feminización, rentabilidad y, finalmente, en cuanto a remuneraciones (Bravo, Sanhueza y Urzúa, 2008; Elfernan, Soto, Colbe y Ramos, 2009; Lara, Meller y Valdés, 2010; Meller, 2010b; Meller, 2010c; Urzúa, 2012; Valdés, Meller y Lara, 2010).

En su conjunto, tales investigaciones dan sustento para afirmar que tanto la carrera como la institución de estudio deben ser tenidas en cuenta para explicar los diferenciales de remuneraciones entre profesionales, no siendo posible asumir una carrera y universidad representativa. Sin embargo, no se ha prestado suficiente atención al hecho de que las características organizacionales e institucionales de las carreras podrían explicar variaciones en las remuneraciones de titulados con iguales atributos personales, como su origen social y rendimiento académico.

Al respecto, las pocas investigaciones que han abordado la materia en Chile (Rappoport et. al., 2004; Meller, 2010c) tienden a tratar metodológicamente las características de distintos niveles como atributos del individuo o, en su defecto, a tratarlas como una estructura multinivel (titulados -carreras- universidades), sin considerar que cada una de ellas posee propiedades que podrían explicar parte de los ingresos de los titulados.

A partir de tales antecedentes, este trabajo tiene como objetivo determinar los principales factores que influyen en las remuneraciones recibidas por universitarios recién titulados, buscando dar evidencia de la existencia de características propias de las carreras que afecten, por sí mismas, las remuneraciones de los titulados. En este sentido, la hipótesis que guía el trabajo indica que parte de tal heterogeneidad salarial se explica por factores organizacionales e institucionales de las mismas tales como la antigüedad, la duración oficial, la selectividad, el retraso y el grado de feminización de la carrera.

La presente investigación tiene la naturaleza de un estudio de caso, pues utiliza datos de una institución en particular para un conjunto de titulados en un periodo específico. Esta institución corresponde a una universidad privada creada a partir de la Ley General de Universidades de 1981, la cual posee un volumen 
intermedio de estudiantes de pregrado (10.000 a 15.000 estudiantes), se encuentra acreditada por cinco años y se clasifica como Universidad de Alta Selectividad, ya que más de la mitad de su matrícula obtiene puntajes PSU por sobre los 601 puntos (Meller, 2010b). A su vez, de acuerdo con informes internos de dicha institución, un 40\% de la matrícula total estudia con Crédito con Aval del Estado (CAE) y un $32 \%$ recibe algún tipo de beca (interna o externa), dando cuenta de la heterogeneidad en su composición de origen.

Dadas sus características, el estudio es novedoso al ser la primera investigación en la materia que ofrece resultados específicos de titulados de una universidad privada no tradicional, que considera un set amplio de carreras de distintas áreas del conocimiento. A su vez, introduce variables clave que no aparecen comúnmente en la literatura, como el efecto salarial de un conjunto de atributos propios de las carreras, especialmente la duración oficial de las mismas, materia de discusión actual en las políticas públicas educativas, cuyas modificaciones en forma aislada podrían tener efectos sobre las remuneraciones de sus titulados, según sugiere el estudio. Todo ello es tratado mediante modelos mixtos o multinivel, los cuales son más exigentes con los datos para establecer diferencias estadísticamente significativas.

\section{Métodos}

La principal fuente de información utilizada es la Encuesta de Inserción Laboral, la cual es parte del Sistema de Seguimiento de Estudiantes y Titulados de dicha universidad y se realiza sistemáticamente desde 2009, aplicándose un año a la mitad de las carreras y, al año siguiente, a la otra mitad. En el presente trabajo se consideraron las tres aplicaciones disponibles al momento de realizado el estudio (2009, 2010, 2011), de modo que las cohortes de titulados corresponden a los años 2008, 2009 y 2010, dependiendo de la carrera) ${ }^{3}$.

3 Existen carreras seguidas dos veces (2009 y 2011) y otras una vez (2010). Se decidió considerar las tres olas de mediciones para no reducir considerablemente el número de casos, limitando a su vez los análisis posibles de efectuar. Revisar en la siguiente subsección referida a los modelos, cómo las regresiones jerárquicas lineales responden a datos de esta naturaleza. 
La recolección de datos de la encuesta es censal y se aplica telefónicamente a la totalidad del universo de alumnos titulados, obteniendo una tasa global de respuesta de $65 \%{ }^{4}$.

Dentro de las consideraciones técnicas para focalizar la investigación en universitarios recién titulados se encuentra que el instrumento cuenta con nutrida información respecto de la inserción laboral y situación laboral actual, no así en cuanto al historial de trabajos realizados por los titulados. Por lo tanto, se consideró solo a aquellos que llevan un año de titulados al momento de aplicarse la encuesta, debido a que el set de preguntas relacionadas con la trayectoria laboral no se incluye para aquellos que llevan tres años de titulados.

Del mismo modo, se consideran solo exestudiantes de carreras diurnas, debido a que la mayoría de los titulados vespertinos ya se encontraba trabajando al momento de titularse (89\%). Situación similar ocurre con los titulados de pedagogías medias, los cuales fueron excluidos debido a que estas corresponden a programas de continuidad para estudiantes ya licenciados de carreras de pregrado, muchos de los cuales podrían haber trabajado luego de titularse de su primera carrera, información con la cual no se cuenta.

Las restricciones anteriormente señaladas fueron impuestas para evitar casos en los que la explicación de la variabilidad de las remuneraciones de titulados se deba a factores no observables en el instrumento, como el canal de obtención del primer trabajo remunerado o el número de meses en encontrarlo, lo cual podría haber producido un sesgo por omisión de variables relevantes y, por ende, una inadecuada especificación de los modelos (Gallego, 2009). En este sentido, corresponde señalar que los resultados se restringen a titulados de carreras de pregrado diurnas de la universidad aludida.

4 La tasa de respuesta varía entre 50\% y 100\% en el rango de las carreras. En resultados no mostrados, se aplicaron test t para observar si existían diferencias estadísticamente significativas entre aquellos que contestaron la encuesta y quienes no, contrastando un conjunto amplio de variables de caracterización con datos para ambos grupos, incluyendo variables sociodemográficas, de origen social y rendimiento universitario. Los resultados indicaron que, en términos generales, no existen mayores diferencias entre aquellos que contestaron la encuesta y quienes no lo hicieron. Esto se refleja en que solo el 9\% de los test aplicados mostró diferencias estadísticamente significativas entre ambos grupos. 
Finalmente, a los datos provenientes de la encuesta se les agregó información sociodemográfica y académica de los exalumnos a partir de los registros del Sistema de Gestión Universitaria (SGU) con que cuenta tal institución.

La variable dependiente de los modelos corresponde al logaritmo natural de la remuneración líquida mensual por hora. Dado que en la encuesta se pregunta por las remuneraciones líquidas a través de diez tramos de ingresos, se calculó el promedio de cada uno de los tramos (Núñez, J. y Gutiérrez, R. 2004a). Luego, cada promedio de las mediciones 2009 y 2010 se reajustó por el IPC a diciembre de 2011. A continuación, se realizó una división para cada caso por el número de horas trabajadas. Finalmente, se aplicó el logaritmo natural al cálculo anterior, para ajustar los datos a una curva de distribución normal y para observar de manera más directa el retorno de los distintos factores explicativos.

Las variables independientes en el ámbito individual (titulados) fueron clasificadas en seis dimensiones: sociodemográficas, de origen social, habilidades cognitivas, capital humano, inserción laboral y trabajo actual 5 .

En cuanto a las características sociodemográficas, se incluyeron sexo (Meller, 2010c; Valdés et. al., 2010; Bravo et. al., 2008), edad y región de procedencia según la comuna del colegio o liceo de egreso de cuarto medio. Estas variables fueron utilizadas como controles.

Dentro de las variables de origen social se incluyó el tipo de colegio (Núñez, J. y Pérez, G., 2007; Elfernan et. al., 2009; Meller, 2010c), si estudió con alguna beca socioeconómica ${ }^{6}$ y si el titulado

5 Se indican variables conceptualmente relevantes incluidas en pruebas de modelos. Como se observa posteriormente en la Tabla 2, no todas ellas se consideraron en los modelos finales expuestos, seleccionándose aquellas de mayor relevancia teórica y poder explicativo.

6 Esto es, si en alguno de los años en que estuvo matriculado obtuvo alguna de las siguientes becas: Beca Internado, Beca Complementaria CAE, Beca al Mérito, Beca Almuerzo, Beca Fotocopia, Beca Plotter, Beca Indígena, Beca Hijo de Profesor, Beca Integración Territorial, Beca Excelencia Académica, Beca Juan Gómez Millas, Beca Municipalidad de Las Condes, Beca Puntajes PSU y/o Beca JUNAEB. 
fue primera generación universitaria ${ }^{7}$ (Blau, P. y Duncan, O., 1967; Bravo et. al., 2008; Zarhi, 2010). A partir de las anteriores variables de origen social (todas dicotómicas) se generó la variable «índice socioeconómico» que las combina en forma aditiva y que puede tomar valores desde 0 (menor) hasta 3 (mayor) ${ }^{8}$.

En habilidades cognitivas se incluyeron variables proxies a partir de indicadores de rendimiento académico universitario ${ }^{9}$. Estas fueron: la nota de titulación (en adelante «nota final»), posición en un ranking relativo dentro de la generación de titulación (en adelante «percentil de nota») y el porcentaje de retraso respecto de la duración teórica de la carrera, indicado como «titulación en tiempo exacto» (titulados en igual duración a la definida en las respectivas mallas de estudio).

En la cuarta dimensión, capital humano, se incluyó si estudió o estudia un posgrado, a qué tipo corresponde y la experiencia laboral, medida a partir del número de trabajos remunerados que ha tenido (Becker, 1993) ${ }^{10}$.

En la quinta dimensión, relativa a las características de la inserción laboral, se incluyó si ha trabajado remuneradamente

7 Sus padres alcanzaron como máximo la educación técnica completa, sin haber asistido nunca a la universidad.

8 El valor «0» indica que el titulado egresó de un liceo municipal, estudió en la universidad con beca socioeconómica y es primera generación universitaria; el valor «1» indica que se dio una de estas situaciones; el valor «2» que se conjugan dos de estas situaciones; y el valor «3» ninguna de ellas.

9 No se consideran las notas de Enseñanza Media (NEM) debido a la evidencia de la variación según tipo de establecimiento de procedencia, observándose que alumnos de colegios particulares pagados tienen notas más altas que alumnos de colegios públicos y subvencionados (Gallegos, Meneses y Parra, 2005). A su vez, pese a su capacidad predictiva (Gallegos et al., 2005; Gallegos y Meneses, 2007; Contreras, Gallegos y Meneses, 2009), no se incluyó el ranking relativo de notas en el colegio debido a que no existe información pública al respecto, y dadas las características de los datos, iniciativas como www.turanking.cl no aplican para la presente investigación. Finalmente, no se incluyó el puntaje de ingreso a la universidad a nivel individual, dado que tales pruebas (PAA/PSU) no están diseñadas para ser comparables a través de las cohortes, y corresponde a una medida individual relativa de rendimiento para un año determinado, existiendo el problema del aumento de cobertura que tiene la prueba año tras año (Núñez, et. al., 2004a).

10 Símil de experiencia utilizada en las Ecuaciones de Mincer. Esto debido a que todos los titulados llevan un año en el mercado del trabajo, por lo que es una constante. A su vez, no se utiliza la variable correspondiente al número de años de educación, ya que, tal como se plantea en la tercera hipótesis, la duración corresponde a un atributo de nivel agregado (carrera). 
desde que se tituló, cuánto se demoró en encontrar el primer trabajo remunerado y cómo lo consiguió, con especial foco en la utilización de redes primarias (familiares o amigos cercanos) (Requena, 1989; Requena, 1991; Calvó-Armengol, 2006; Agencia Nacional de Evaluación de la Calidad y Acreditación, 2008).

Finalmente, se incluyeron como variables de control algunos factores relacionados con el trabajo actual como el tipo de labor, el sector, la jornada laboral y la remuneración líquida mensual. Esta última fue la base para construir la variable dependiente.

A nivel agregado (carreras), los principales predictores fueron el grado de feminización (porcentaje de mujeres), la selectividad de la carrera (puntaje promedio en la prueba de ingreso) ${ }^{11}$, duración oficial (número de semestres declarados en el plan de estudio), retraso (porcentaje promedio entre duración teórica y real) y antigüedad (número de años desde la creación de la carrera en la universidad). Estas variables fueron utilizadas para probar la existencia de características propias de las carreras que pudieran explicar parte de la variabilidad de las remuneraciones entre titulados (Rappoport, Meller y Lara, 2010; Meller, 2010b; Meller, 2010c; Elfernan et. al., 2009; Lara et. al., 2010).

Se utilizaron regresiones jerárquicas lineales como técnica estadística, teniendo como variable dependiente el logaritmo natural de la remuneración líquida mensual por hora.

Este tipo de regresiones, también conocidas como modelos mixtos o multinivel, son útiles cuando no es posible asumir el supuesto MICO de independencia de los errores, lo cual ocurre en este caso debido a que los titulados se encuentran agrupados en unidades de anidamiento, coexistendo dos unidades aleatorias (individuos y carreras).

11 Se considera el puntaje promedio como medida de selectividad en vez del puntaje de corte debido a que, para los años de ingreso de los titulados observados, la universidad bajo análisis no pertenecía a un sistema integrado de admisión junto con otras instituciones, de modo que tal puntaje de corte era preestablecido por igual para todas las carreras, exceptuando Medicina, Derecho y Psicología con puntajes superiores exigidos para el ingreso. Así, tal puntaje hubiese entregado poca variabilidad para los modelos y escaso poder de discriminación en cuanto al rendimiento general de entrada para una carrera y otra. 
Utilizando el software HLM 6.0 fue posible identificar la magnitud con la que las características estructurales de las unidades agregadas explican las diferencias en la variable de resultado entre los individuos, al permitir que la (ln) remuneración líquida mensual por hora varíe entre las diferentes carreras y que sus efectos (aleatorizados) sean explicados por predictores propios de la unidad de anidamiento. De esta forma se obtienen coeficientes «limpios» del efecto de diferencias en características individuales de los titulados sobre las remuneraciones (Bryk y Raudenbush, 1992) ${ }^{12}$.

De acuerdo con lo señalado, las ecuaciones de regresión son: (1)(Ln) Ingreso $_{i j}=\beta_{0 j}+\beta_{1 j}\left(\right.$ controles $\left._{i j}\right)+e_{i j}$

(2) $\beta_{0 j}=\gamma_{00}+\beta_{01}\left(\right.$ porc_mujeres $\left._{j}\right)+\beta_{02}\left(\right.$ antiguedad $\left._{j}\right)+\beta_{03}\left(\right.$ selectividad $\left._{j}\right)+\beta_{04}\left(\right.$ duración $\left._{j}\right)+$ $+\beta_{05}\left(\right.$ porc_retraso $\left._{j}\right)+r_{0 j}$

Donde:

$i=$ titulado

$j=$ carrera

En la ecuación (1), la variable dependiente ((Ln) Ingreso) corresponde al logaritmo natural de la remuneración líquida mensual por hora del titulado «i» en la carrera «j». Por su parte, en «controles» se resumen las variables de control a nivel 1 , en donde se incluyeron características sociodemográficas, de capital humano, laborales, habilidades cognitivas, redes primarias y origen social. A esto se le incorporó un término de error aleatorizado a nivel $1\left(e_{i j}\right)$, bajo el supuesto de distribución normal, con media (0) y varianza constante a nivel $1\left(\sigma^{2}\right)$.

En la ecuación (2), la variable dependiente $\left(\beta_{0 j}\right)$ es el término de intercepto de la ecuación (1), correspondiendo al promedio en

12 Tal como fue señalado, se consideraron carreras con dos mediciones y otras con una sola. De todas formas, los modelos jerárquicos permiten diseños «desbalanceados» (la cantidad de unidades dentro de cada grupo es variable), ya que la estimación de cada efecto aleatorio (estimación empírica bayesiana) corresponde a un promedio ponderado de la media grupal y la media total, donde el peso asignado a cada elemento es proporcional al nivel de variación de las observaciones, lo que a su vez está determinado tanto por la varianza de las respuestas acerca de los salarios dentro de cada carrera, como por el número de observaciones consideradas dentro de cada caso. De este modo, el efecto aleatorio de las carreras con más observaciones es estimado con un menor nivel de incertidumbre, comparado con carreras con menor cantidad de observaciones. 
la carrera «j» de las remuneraciones líquidas mensuales por hora, el cual es condicional a la gran media de remuneraciones de todas las carreras $\left(\gamma_{00}\right)$, y las variables independientes a nivel de las unidades de anidamiento: porcentaje de mujeres en la carrera («porc_mujeres»), número de años desde la creación de la carrera («antigüedad»), promedio en la prueba de ingreso a la universidad («selectividad»), duración oficial de la carrera («duración») y porcentaje promedio de retraso en la carrera («porc_retraso»), junto con un término de error a nivel $2\left(r_{0 j}\right)$, bajo el supuesto de distribución normal, con media (0) y varianza constante a nivel $2\left(\tau_{00}\right)^{13}$.

\section{Resultados}

\subsection{Descripción de las carreras}

Dado que el estudio plantea la existencia de características a nivel agregado que explicarían parte de las diferencias en las remuneraciones de los titulados, la Tabla 1 expone el promedio de remuneraciones líquidas por hora de cada carrera y un set de variables explicativas de tal nivel de agregación.

Tal como señala la evidencia previa (Elfernan et. al., 2009; Urzúa, 2012; Lara et. al., 2010) existen importantes diferencias de remuneraciones de acuerdo con la carrera estudiada. Exhibida en forma de ranking, la Tabla 1 muestra que la carrera mejor remunerada (Medicina) presenta ingresos promedio por hora cercanos a tres veces la carrera con menor remuneración promedio (Pedagogía Básica). A su vez, coherente con la evidencia empírica (Meller, 2010b; Elfernan et. al., 2009), las carreras tradicionales como Medicina, las Ingenierías Civiles e Ingeniería Comercial se ubican en las primeras posiciones del ranking de ingresos promedio por hora ${ }^{14}$.

13 Todas las variables cuantitativas fueron centradas en sus medias: edad, meses en encontrar el primer trabajo remunerado e índice socioeconómico. Esto a fin de entregar interpretaciones más razonables de los coeficientes e intercepto, lo cual no afecta la calidad del modelo de ajuste (Gibaja, 2008).

14 En el caso de la carrera de Derecho, es preciso considerar que el grado que entrega la universidad es la Licenciatura en Ciencias Sociales y Jurídicas, pues el título lo entrega la Excelentísima Corte Suprema. Dado que se consideran aquellos titulados que llevan un año en el mercado del trabajo, es posible que un porcentaje relevante no haya realizado aún el juramento y, sin el título, las remuneraciones pueden ser inferiores a aquellos que sí cuentan con este. 
Por otra parte, la Tabla 1 muestra la importante heterogeneidad en las variables agregadas a nivel de carreras, tal como señalan Lara, Meller y Valdés (2010). Así, el grado de feminización varía entre 0\% (Ingeniería Civil en Informática y Telecomunicaciones) y 100\% (Pedagogía en Educación Parvularia) y el grado de selectividad varía entre 518 puntos promedio en Pedagogía en Educación Parvularia en las pruebas de ingreso y 696 puntos promedio en Medicina.

También existen diferencias ostensibles en la duración oficial de los respectivos planes de estudio: mientras las carreras tales como Historia, las Pedagogías y Literatura Creativa contemplan una duración teórica de ocho semestres, la duración oficial en Medicina es de 14 semestres. A su vez, la antigüedad de las carreras fluctúa entre 28 años (Ingeniería Comercial, Derecho y Psicología) y un conjunto de carreras abiertas en 2002 (nueve años). En cuanto al nivel de retraso, la duración teórica y la real varía entre un promedio de $1 \%$ (Medicina) y 62\% (Derecho).

Dada la naturaleza de la hipótesis planteada se generaron correlaciones parciales entre la remuneración líquida por hora promedio en cada carrera y cada una de las variables agregadas. Tal como se aprecia, existe una relación negativa moderada entre remuneración promedio en la carrera y el porcentaje de mujeres en la misma $(-0,48)^{15}$, una relación positiva moderada entre remuneración promedio y selectividad $(0,51)$ y una relación positiva muy alta entre remuneración promedio y duración oficial de la carrera $(0,81)$.

Los resultados preliminares anteriormente expuestos permiten aventurar la existencia de una heterogeneidad estructural, en donde las características propias de las carreras ayudarían a explicar parte de la variabilidad de las remuneraciones de los titulados.

15 Coeficiente idéntico al observado por Valdés, Meller y Lara (2010). 


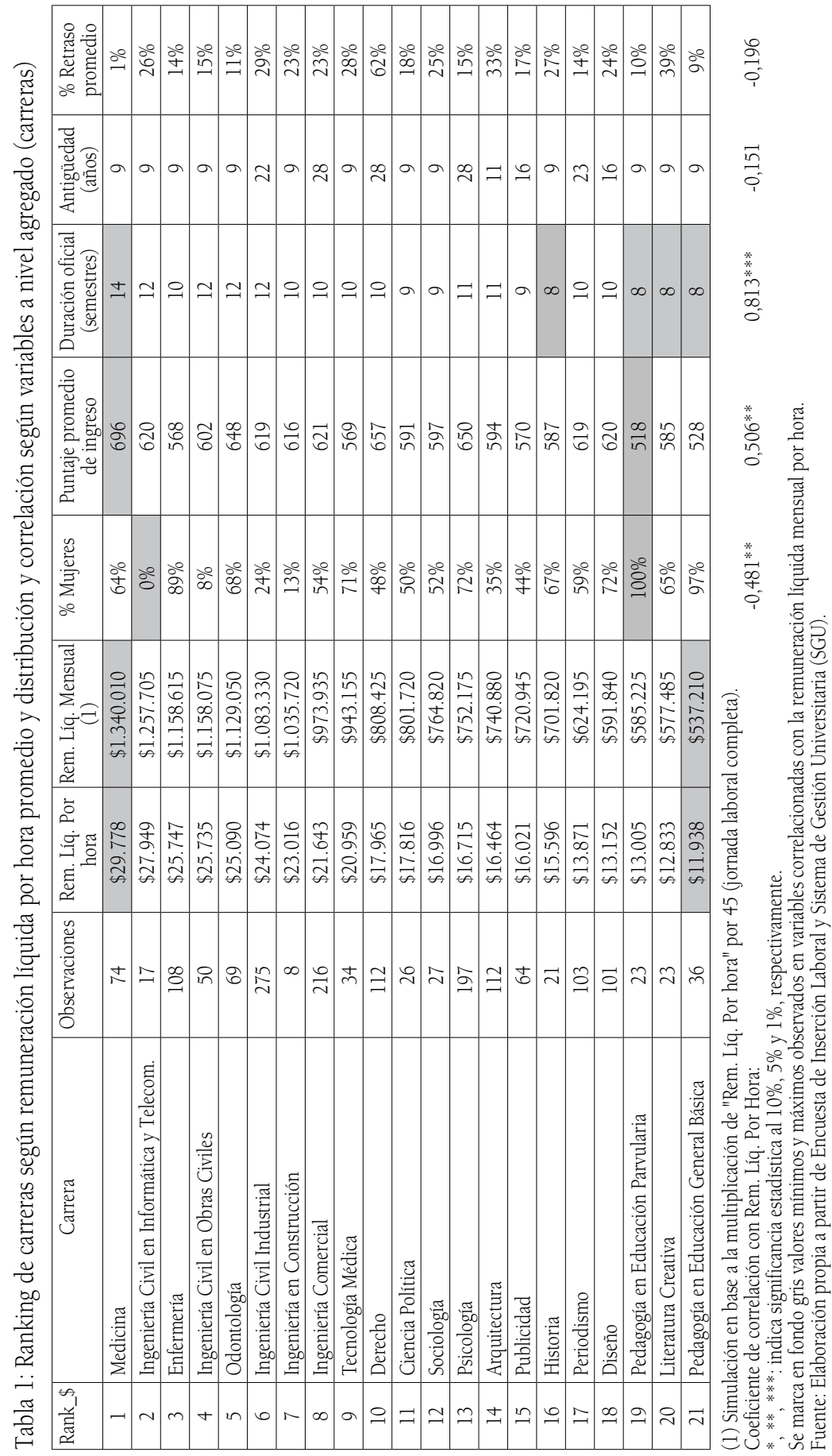




\subsection{Análisis de regresión}

Para responder adecuadamente a la pregunta de si existen características de las carreras que expliquen por sí mismas parte de la variabilidad de las remuneraciones una vez controlados los atributos de los titulados, a continuación se expone un conjunto de modelos de regresiones jerárquicas lineales.

Tal como fue señalado, este tipo de modelos resulta útil cuando los casos están agrupados en unidades de información más amplias y se consideran medidas tanto en el nivel más bajo (titulados) como en el nivel más alto (carreras) (Pardo, Ruiz y San Martín, 2007).

El primer modelo en la Tabla 2, conocido como nulo o no condicional, se encuentra exento de variables explicativas, pues en su versión reducida solo depende de la gran media de remuneraciones $\left(\gamma_{00}\right)$ y de dos fuentes de variación: intragrupo $\left(e_{i j}\right)$ y entre grupos $\left(r_{0 j}\right)$.

El modelo nulo indica que las remuneraciones varían significativamente a través de las carreras, información extraída a partir de la aleatorización de la varianza del término de error a nivel $2\left(\tau_{00}\right)$, cuyo valor $(0,08)$ es significativo con un nivel de confianza de $99 \%$.

Al mismo tiempo, en el apartado de efectos aleatorios (parte inferior de la tabla) se incluye la correlación intraclase, medida que indica el peso de la heterogeneidad del grupo, pues compara el grado de variabilidad existente entre las distintas carreras en relación con la variabilidad total ${ }^{16}$. En el caso del modelo nulo, la correlación intraclase es de $37 \%$, lo que significa que más de un tercio de lo no explicado se debe a características propias de las carreras. Esto justifica la utilización de este tipo de regresiones multinivel, pues supone la presencia de heterogeneidad estructural, existiendo

16 Este valor fluctúa entre $0 \%$ y $100 \%$. Un valor de 100\% indicaría que toda la variabilidad se debe al factor, es decir, a la diferencia entre las carreras (lo que solo ocurriría cuando en todos los titulados de una misma carrera se diera la misma remuneración y las carreras presentaran diferentes promedios). Un valor de $0 \%$ indicaría que el factor no contribuye a explicar la variabilidad de la remuneración, es decir, toda la varianza tiene su origen en las diferencias existentes dentro de cada carrera (lo que solo ocurriría cuando en todas las carreras se diera la misma remuneración promedio). 
atributos observables e inobservables de las carreras que afectarían las remuneraciones de los titulados ${ }^{17}$.

Esta evidencia del peso de las carreras respecto de la variación de las remuneraciones de los titulados es consistente con lo encontrado por Rappoport et. al. (2010), pues incluso en modelos que consideran carreras anidadas en universidades, se pone de manifiesto que los atributos de las primeras explican más del doble de la varianza del salario que las características de las segundas. Así, la jerarquía explicativa estaría dada por atributos de los titulados, luego por características de las carreras y finalmente por cualidades de las universidades.

Conocida la correlación intraclase (modelo 1, Tabla 2), los siguientes corresponden a modelos condicionales, pues poseen variables explicativas de nivel 1: las remuneraciones de los titulados dependen de un conjunto de variables propias de los titulados y de otros atributos organizacionales e institucionales propios de las carreras.

Centrándose en el efecto de las características de los titulados sobre los ingresos, se observa en forma robusta que no existe un efecto salarial neto de la titulación en tiempo exacto, como tampoco del índice socioeconómico del titulado, ni un retorno de las redes primarias (haber obtenido el primer trabajo remunerado por intermedio de un familiar o un amigo cercano, en comparación con los restantes canales de obtención de empleo) ${ }^{18}$.

Al mismo tiempo, la Tabla 2 permite apreciar que a mayor edad, mayores son las remuneraciones ( $2 \%$ extra por cada año adicional) y que existe una brecha salarial de género de un $7,2 \%$ en contra de las

17 De esta forma, se detecta la no independencia de los errores, pues los casos están relacionados por un contexto de pertenencia, a saber, la carrera estudiada.

18 En resultados no exhibidos se probaron modelos con variables alterativas en cada una de tales dimensiones. Así, se utilizó también el promedio final de nota y el ranking de nota relativo en la universidad como medidas de rendimiento académico; la educación de los padres; tenencia de becas socioeconómicas y tipo de colegio; el origen social y el set completo de canales de obtención de empleo como variables dummy (dejando como categoría de referencia el canal más rentable) y medidas de redes primarias. Ninguna de tales combinaciones mostró retornos salariales, controlando las restantes variables. 
mujeres; por otro lado, aquellos que tardaron más en encontrar su primer trabajo remunerado obtienen menores remuneraciones (1,9\% menos por cada mes adicional en encontrar trabajo), aquellos que se desempeñan en el sector público obtienen cerca de un $6 \%$ adicional de remuneración, comparado con aquellos que lo hacen en otros sectores (privado o privado sin fines de lucro) y que los titulados con contrato indefinido obtienen sobre un $7 \%$ adicional de ingresos comparado con otras situaciones contractuales.

En su conjunto, los modelos completos ( 2 al 7) permiten reducir en torno a un $13 \%$ la varianza no explicada a nivel $1\left(\sigma^{2}\right)$ con respecto al modelo nulo.

\subsubsection{El retorno de las carreras}

Tal como señala la literatura (Bravo et. al., 2008; Elfernan et. al., 2009; Lara et. al., 2010; Meller, 2010b; Meller, 2010c; Urzúa, 2012; Valdés et. al., 2010) y la evidencia específica expuesta (ver Tabla 1), existe una gran heterogeneidad en las carreras en aspectos tales como el grado de selectividad, la duración de los planes de estudio y su rentabilidad. A partir de tal constatación, se buscó identificar en qué medida los atributos institucionales y organizacionales tales como los expuestos generan diferencias en las remuneraciones de los titulados, independientemente de las características propias de estos, como su rendimiento académico universitario, el origen social que poseen los titulados o el sector donde trabajan.

En la presente sección se pone a prueba la hipótesis planteada mediante la aplicación de regresiones jerárquicas lineales. Tal como fue expuesto (ver ecuación número 1, en sección 2), se revisa en qué medida la variabilidad del promedio de remuneraciones entre una carrera y otra se explica por los atributos propios de la unidad de anidamiento, controlado por las características propias de los titulados.

Para el modelamiento en la hipótesis en cuestión se enfrentó la restricción de contar con solo 21 casos en el nivel agregado, correspondientes al total de carreras en la institución bajo análisis. Dada la recomendación de no incluir más de un parámetro por 
cada 10 observaciones (Bryk et. al., 1992), se decidió implementar un modelo por cada variable independiente de nivel $2^{19}$. El último de los modelos (número 7) viola tal recomendación, incluyendo en una sola regresión todas las variables de nivel 2 que aparecen como estadísticamente significativas, para revisar la robustez de estas.

El conjunto de los modelos 2 al 6 expuestos en la Tabla 2 indica la presencia de tres variables propias de las carreras que ayudan a explicar las diferencias en las remuneraciones de los titulados: el porcentaje de mujeres, el promedio en puntaje de ingreso a la universidad y la duración oficial de la carrera.

Así, en el segundo modelo se observa que a mayor porcentaje de mujeres en la carrera, menores remuneraciones $(-0,005)$. De esta forma, por ejemplo, al pasar de una carrera completamente masculinizada como Ingeniería Civil Informática ( $0 \%$ de mujeres) a otra totalmente feminizada como Educación Parvularia (100\% mujeres) la reducción de las remuneraciones es cercana a un 50\%, efecto que resulta independiente de las características observadas de los titulados ${ }^{20}$. Este resultado es consistente con lo reportado por Meller (2010c), quien encuentra un castigo salarial para los titulados en carreras con alta participación femenina.

Ante la evidencia encontrada, se probó si la brecha salarial de género (en torno a un 7\% en contra de las mujeres) es explicada o no por el porcentaje de mujeres en la carrera. En resultados no mostrados, se rechaza que la brecha salarial de género (nivel 1) varíe según el grado de feminización (nivel 2) ${ }^{21}$. Así, se descarta la teoría de la «escalera de vidrio» (Budig, 2002) que indica que hombres en ocupaciones feminizadas logran sortear parte de las penalidades de la composición de género, aprovechándose de ventajas estructurales no directamente observables (Cohen y Huffman, 2003). De este modo, tanto en titulados hombres como mujeres existe una pérdida

19 Esto debido a que, junto con la variable explicativa, cada modelo contará con un término de intercepto, que corresponde a la gran media salarial de todas las carreras.

20 Dado que el coeficiente marca $-0,005$, cada punto porcentual de aumento en la cantidad de mujeres en la carrera implica una reducción en 0,5\% de las remuneraciones. Se asume un efecto lineal del porcentaje de mujeres sobre las remuneraciones.

21 Coeficiente 0,001295 no significativo. 
de remuneraciones por encontrarse en una carrera feminizada, manteniéndose la brecha salarial de género.

A su vez, el segundo modelo permite mostrar evidencia de un importante efecto de composición de género. El coeficiente obtenido $(0,067)^{22}$ revela que la brecha salarial de género desfavorable para las mujeres observada al interior de las carreras es muy superior al efecto que tiene el porcentaje de mujeres sobre las diferencias en remuneraciones entre las carreras, dando cuenta de un mayor efecto within que between.

Por otra parte, el cuarto modelo prueba el efecto de la selectividad de la carrera sobre las remuneraciones de los titulados ${ }^{23}$. De acuerdo con lo observado, existe un efecto positivo y significativo del puntaje promedio de ingreso a la carrera sobre las remuneraciones, aumentando en 0,3\% los ingresos por cada punto adicional en el promedio. Así, por ejemplo, titulados de carreras selectivas con puntajes promedio levemente inferiores a 700 puntos (como Medicina), logran ingresos cerca de un 30\% superior a titulados de carreras menos selectivas, que presentan puntajes promedio levemente inferiores a 600 puntos (como Literatura Creativa), lo cual es independiente del desempeño universitario del titulado, su origen y otros atributos individuales observados.

Del mismo modo, aparece un fuerte efecto salarial en cuanto a la duración oficial de la carrera. De acuerdo con lo expuesto en el quinto modelo, por cada semestre adicional de duración los titulados presentan un 12,6\% extra de remuneraciones. De esta forma, quienes estudiaron carreras de larga duración como las Ingenierías Civiles (12 semestres) reciben ingresos cercanos a un $25 \%$ superior a aquellos que estudiaron carreras de menor duración, como Periodismo o Diseño (10 semestres), incluso luego de controlar por sus atributos individuales ${ }^{24}$. Este resultado es similar a lo encontrado por Meller

22 Resta que corresponde a $-0,005--0,072$.

23 Selectividad medida como promedio en la PAA o PSU.

24 Modelo de gran bondad de ajuste, pues permite reducir en un $61 \%$ la varianza no explicada de las remuneraciones entre las carreras $\left(\tau_{00}\right)$. 
(2010c), quien muestra un aumento de $21 \%$ en remuneraciones por cada año adicional en la duración teórica de la carrera.

En el séptimo y último modelo se incluyen las tres variables propias de las carreras que aparecen como estadísticamente significativas: el porcentaje de mujeres, la selectividad y la duración oficial. Pese a indicios de multicolinealidad entre ellas ${ }^{25}$, y a los reducidos grados de libertad, se reafirma el efecto del número de semestres de la carrera sobre las remuneraciones de los titulados, mostrando ingresos $12,9 \%$ superiores por cada semestre adicional definido por la carrera.

Dada la evidencia expuesta, se acepta la hipótesis planteada. Así, como aparece en el modelo nulo, más de un tercio de las diferencias no explicadas de remuneraciones entre titulados se debe a las características de las carreras, demostrando la existencia de heterogeneidad estructural ${ }^{26}$. Esta heterogeneidad se debe en gran medida al grado de feminización de la carrera, su selectividad y el número de semestres predefinidos; variables propias de las carreras que ayudan a explicar las diferencias en las remuneraciones entre los titulados. Esto último, la duración oficial, aparece como un resultado robusto entre los modelos, dando signos de un premio por parte del mercado del trabajo para quienes estudien carreras de larga duración, incluso luego de controlar el grado de selectividad, porcentaje de mujeres y atributos propios de los titulados, como su rendimiento académico, origen social y factores laborales.

25 Correlación de .73 entre duración oficial y selectividad de la carrera.

26 Esto acompañado de estimaciones de fiabilidad superiores a 0,8 en todos los modelos mixtos, indicando que los coeficientes aleatorizados no pueden considerarse como fijos (iguales entre los grupos). 


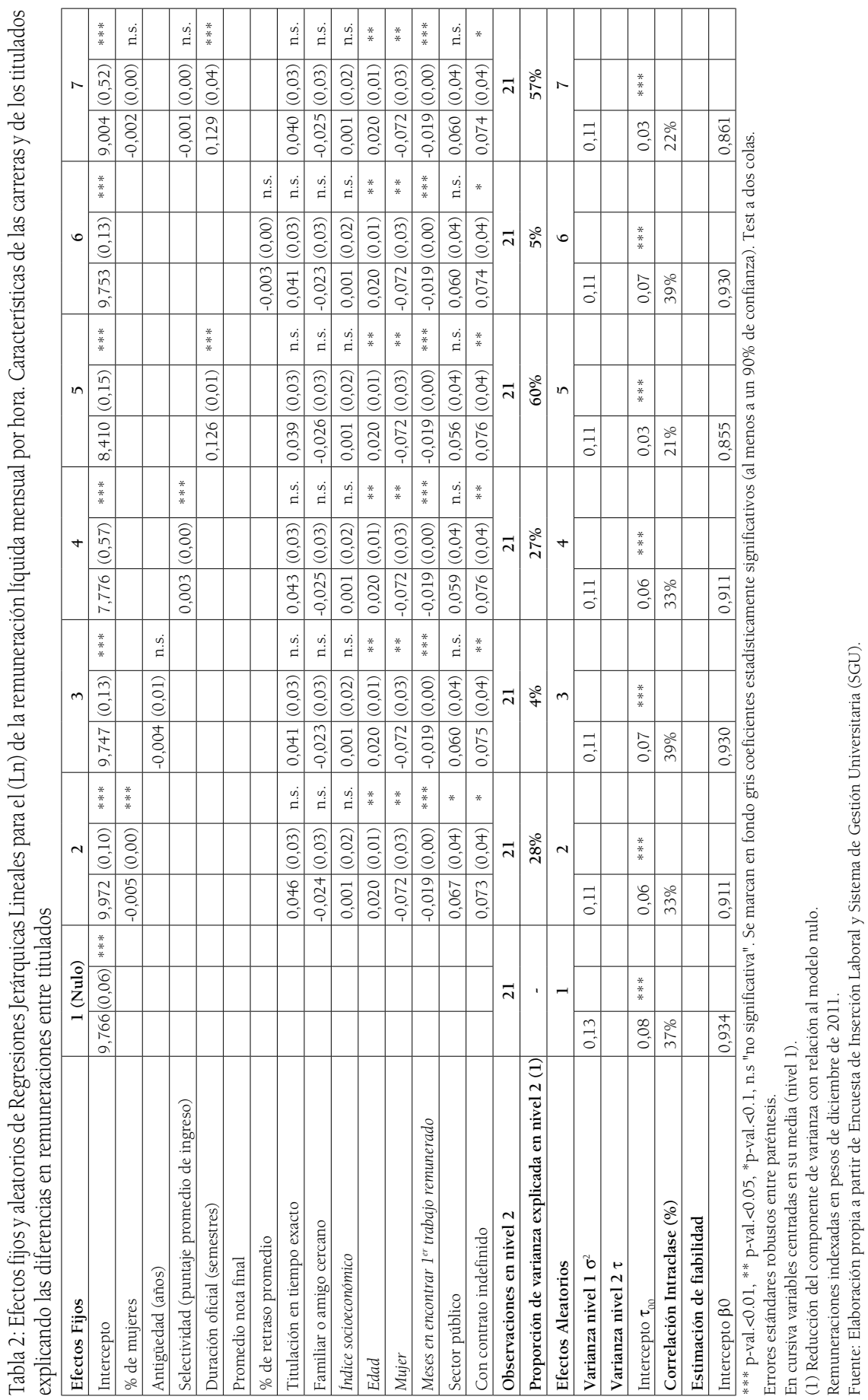




\section{Discusión}

El presente trabajo ha tenido como propósito ampliar la evidencia existente en Chile respecto de los factores que inciden en las remuneraciones de universitarios recién incorporados al mercado del trabajo, a partir de un estudio de caso de titulados de una universidad específica. Esto en el marco de un diagnóstico generalizado acerca del déficit de información disponible para los diversos agentes del sistema, especialmente para los estudiantes y policymakers (Brunner, 2004; OCDE, 2009).

Pese al auge de estudios en la materia en los últimos años, poca ha sido la contribución de las universidades privadas no tradicionales, a pesar de que hoy en día corresponden al principal oferente de matrícula en todo el Sistema de Educación Superior (SIES, 2012a).

En general, los estudios como el presente que controlan por un set amplio de variables relevantes, especialmente las vinculadas con el origen social y con el desempeño universitario, se han concentrado en universidades miembro del Consejo de Rectores de Chile (CRUCH), focalizándose en un grupo reducido de carreras tradicionales (Núñez, J. y Risco, C., 2004b; Núñez et. al., 2004a; Bravo et. al., 2008; Urzúa, 2012). La investigación realizada pretendió ampliar el campo, reportando resultados salariales de una universidad privada, considerando un conjunto amplio de carreras en distintas áreas del conocimiento y añadiendo a su vez variables clave en discusiones actuales de políticas públicas en educación superior, como el efecto salarial de la duración teórica de las carreras.

Los principales resultados del trabajo se relacionan con la incidencia observada de ciertas características de las carreras sobre las remuneraciones de universitarios recién titulados. De esta forma se obtuvo que más de un tercio de la variabilidad de los ingresos de los titulados se debe a atributos propios de la carrera, lo cual indica la no conveniencia de realizar estudios en la materia que omitan información acerca de tal contexto de pertenencia. Así, dada la heterogeneidad estructural observada, se justifica el uso de un modelo multinivel, con titulados (nivel 1) anidados en carreras 
(nivel 2), en donde cada nivel aporta factores explicativos respecto de las variaciones en los ingresos de los titulados.

Dentro de los hallazgos puntuales gracias al uso de modelos mixtos, con información de las carreras explicando las variaciones en el promedio de las remuneraciones, se cuenta que las carreras feminizadas -con un alto porcentaje de mujeres-y las carreras menos selectivas -con un bajo puntaje promedio de ingreso- se relacionan negativamente con las remuneraciones de los titulados, incluso luego de controlar un conjunto de características individuales como el género, el origen social y el desempeño universitario, entre otros.

Tales efectos observados podrían contradecir en parte la teoría del capital humano (Becker, 1983; Becker, 1993), pues los ingresos no solo estarían determinados por las distintas inversiones racionales realizadas por los sujetos, sino también por un contexto exterior que actúa con fuerza sobre ellos, como la selectividad de la carrera o el componente agregado de género. Al respecto, futuros estudios podrían indagar en la existencia de discriminación social o endógena para explicar la asociación entre una alta feminización y las bajas remuneraciones promedio, en donde las mujeres sean impulsadas con mayor propensión hacia carreras más compatibles con estereotipos sociales de su rol dual -en el hogar y el mercado laboral-, guiándose hacia profesiones con mayor grado de flexibilidad, especialmente en la jornada de trabajo y con menores penalidades futuras a la discontinuidad en sus trayectorias laborales (Meller, 2010c).

Del mismo modo, se obtuvo un importante efecto de la duración oficial de la carrera sobre las remuneraciones. Así, las mayores demoras teóricas de la carrera impactarían positivamente en los ingresos, obteniendo sueldos 13\% superiores por cada semestre adicional de duración, controlado por las características individuales y propias de las carreras.

Tal resultado es relevante en el contexto actual de discusión acerca de la duración de las carreras, el que ha incluido lineamientos hechos por el Ministerio de Educación para acortarlas (El Mercurio, 2012) y argumentos entregados por las autoridades universitarias 
respecto del bajo nivel de competencias con que ingresan los estudiantes, que las obliga a dedicar tiempo para la nivelación, dificultando una reducción en el número de semestres oficiales ( $\mathrm{La}$ Segunda, 2012).

Dada la evidencia obtenida, se sugiere incluir en la discusión el efecto salarial de la duración de la carrera, pues el número de semestres teóricos que implica estudiar una de ellas tiene repercusiones en el mercado del trabajo, el cual asigna valor a las carreras largas. Esta asignación podría explicarse a factores tales como la complejidad asumida respecto de sus programas y/o habilidades adquiridas a lo largo de los planes de estudios que requieren mayor extensión, lo cual se refleja en carreras que poseen sistemáticamente mayor cantidad de créditos totales y créditos promedio por semestre, los que cuantifican la carga de tiempo (presencial y no presencial) que un alumno promedio requiere para lograr los aprendizajes esperados.

De esta forma, un acortamiento desregulado de ciertas carreras mediante decisiones aisladas por parte de las propias instituciones podría ser interpretado en el mercado laboral como un signo del empeoramiento o de la reducción de las habilidades adquiridas en los profesionales de tales carreras, pudiendo implicar menores remuneraciones. Esto se sostiene especialmente debido a la fuerte correlación demostrada entre la duración teórica y la selectividad, en donde el primer atributo funcionaría como una señal del segundo, gracias a su naturaleza estandarizada y de fácil verificación.

Al respecto, resulta iluminador que para la inserción al submercado laboral de la administración pública los requisitos para el ingreso a sus diversos estamentos y grados consideran y utilizan como mecanismo de jerarquización las distintas duraciones teóricas de las carreras, más que las exigencias individuales de desempeño académico, como podrían ser la concentración de notas o el ranking en la generación. Así, por ejemplo, en la planta de profesionales de la Subsecretaría del Ministerio de Bienes Nacionales se exige para ingresar a los cargos comprendidos entre los grados $4^{\circ}$ al $7^{\circ}$ contar con un título profesional de una carrera de a lo menos 10 semestres, mientras que para el acceso a los cargos de grados $8^{\circ}$ al $12^{\circ}$ se requiere 
un título profesional de una carrera de a lo menos ocho semestres de duración, y para los cargos entre los grados $13^{\circ}$ y el $15^{\circ}$ se solicita estar en posesión de un título profesional de una carrera de a lo menos seis semestres (Neira y Núñez, 2006).

Pese a la naturaleza del caso del presente estudio, la composición de las carreras observada en la universidad bajo análisis parece ser el reflejo de un patrón propio del sistema universitario. Así, es generalizada la presencia de carreras feminizadas como Educación Básica y otras masculinizadas como Ingeniería Civil (SIES, 2009a); existen carreras con un nivel de selectividad sistemáticamente mayor como Derecho y otras de menor puntaje promedio de ingreso como Educación Parvularia (Meller 2010b) y carreras como Medicina presentan persistentemente mayor duración teórica que otras como Periodismo (SIES, 2009b). Así, es plausible hipotetizar que los efectos aquí observados de variables propias de las carreras explicando la variabilidad de las remuneraciones de los titulados podrían ser parte de un fenómeno extendido entre universidades afines a esta en cuanto a la composición del estudiantado (similares características propias de los titulados), más que resultados idiosincráticos de una sola institución.

Dado lo anteriormente expuesto, estudios a futuro debieran ampliar el número de carreras bajo análisis multinivel, reduciendo las limitaciones de contar con pocos casos a nivel agregado. Esto especialmente considerando que aun los ingresos promedio siguen variando significativamente a través de las carreras, incluso luego de incluir como predictores el porcentaje de mujeres, la selectividad y la duración teórica de la carrera, lo cual da cuenta de que variables estructurales no exploradas impactarían en las remuneraciones de universitarios recién titulados.

A partir de lo señalado, estudios posteriores podrían considerar el nivel de demanda por profesionales de distintas carreras en sus respectivos submercados laborales, o bien, variables de aproximación a la calidad de la carrera que pudieran generar diferencias en las remuneraciones incluso dentro de una misma institución, con titulados de similares características observadas a nivel individual. 
Al respecto, desgraciadamente el principal indicador que debiese certificar la calidad de los procesos internos y sus resultados -la acreditación o no del programa y el número de años otorgadoscorresponde a un proceso voluntario ${ }^{27}$, con un considerable margen de amplitud y discrecionalidad (OCDE, 2009). De esta manera, al interior de una misma institución, hoy en día no sería evidente que una carrera acreditada pueda asumirse como un programa necesariamente de mayor calidad que otra no sometida a tal proceso de revisión, o incluso que otra carrera acreditada por un menor periodo, habiendo sido evaluada por la misma agencia acreditadora u otra, organismos que a su vez, no presentan igual oferta para todos los campos disciplinarios.

Del mismo modo, otras investigaciones podrían considerar efectos más amplios sobre la inserción laboral de las variables observadas de los titulados y de las carreras, más allá del impacto de estas sobre las remuneraciones (variable dependiente escogida para el estudio). Así, resultaría relevante explorar los factores individuales y agregados que influyen sobre el tiempo en encontrar un trabajo remunerado. Se abre entonces como pregunta de investigación potencial, si las carreras feminizadas aseguran o no -tanto para hombres como para mujeres- mayores posibilidades de encontrar rápidamente un trabajo remunerado, más allá del salario percibido.

A partir de la evidencia de importantes efectos de niveles agregados sobre las remuneraciones de universitarios recién titulados, también se sugiere avanzar hacia investigaciones que incluyan un tercer nivel de agregación: la universidad de procedencia. Esto debido a que existen atributos de las universidades que podrían ayudar a explicar las variaciones entre los titulados de iguales características y una misma carrera de estudio. Dentro de estas características se encuentra la pertenencia o no al Consejo de Rectores de Chile $(\mathrm{CRUCH})$, si corresponde a una universidad estatal, si pertenece al Sistema Único de Admisión, el número de años de acreditación institucional, número de sedes con que cuenta y su antigüedad, entre

27 Exceptuando para Pedagogías y Medicina. 
otras $^{28}$. A su vez, futuros estudios podrían desarrollarse comparando efectos de atributos individuales para una misma carrera en distintas instituciones.

Para dar el paso de estudios de caso como el presente hacia investigaciones de mayor amplitud como las señaladas, se requeriría de un esfuerzo colaborativo por parte de las instituciones para poner a disposición mutua los datos individualizados que actualmente no son públicos, como el origen socioeconómico de cada titulado, su desempeño universitario y las remuneraciones, entre otros aspectos. Dada la dificultad de una coordinación de ese tipo, se sugiere que el Ministerio de Educación ponga a disposición de los distintos agentes del sistema tales datos, con los que actualmente cuenta, con los debidos resguardos de uso de la información y confidencialidad (sin identificación de RUT ni nombres, por ejemplo). Los beneficios de una iniciativa de este tipo se han visto reflejados en los prolíferos trabajos desarrollados por el Centro de Investigación Avanzada en Educación, quienes al contar con tales datos dieron lugar a múltiples estudios reunidos en el libro Carreras universitarias: rentabilidad, selectividad y discriminación (Lara et. al., 2010; Meller et. al., 2010a; Meller, 2010b; Meller, 2010c; Valdés et. al., 2010).

Finalmente, se recomienda avanzar hacia un Sistema Nacional de Seguimiento de Estudiantes y Titulados. Esto debido a que existe información cualitativa relevante respecto de la progresión de los alumnos y su inserción en el mercado del trabajo que solo es posible obtener mediante la aplicación de encuestas en las distintas etapas del «ciclo de vida» de un estudiante. Experiencias como la realizada por Corea del Sur debieran ser consideradas como un referente, que a través del College Graduate Employment Survey (CGES), dependiente del Ministerio de Educación, ha avanzado hacia la recolección a nivel nacional de información amplia como el porcentaje de egresados que logra obtener un empleo después de su graduación, la relación

28 Para revisión de variables de aproximación a la calidad de la institución, considerar Rumberger R. y S. Thomas, (1993) «The economic returns to college quality, major, and performance» en Economics of Education Review, quienes incluyen variables a nivel de anidamiento como el porcentaje de postulantes aceptados, porcentaje de docentes a tiempo completo o porcentaje de docentes con un doctorado. 
entre la carrera estudiada y el trabajo obtenido y la satisfacción con la carrera profesional emprendida a la fecha, entre otros aspectos (Elacqua, 2004).

\section{Referencias}

Agencia Nacional de Evaluación de la Calidad y Acreditación (ANECA), (2008) Informe graduados. Titulados universitarios y mercado laboral. Proyecto REFLEX. Disponible en: https://observatorio.um.es/observatorio/ observatorio.contenidos. mostrarinformacion. do? apartado $=20 \&$ men $\mathrm{u}=$ otrosinformes

Becker, G., (1983) El capital humano. Un análisis teórico y empírico referido fundamentalmente a la educación. Madrid, Alianza Editorial.

Becker, G., (1993) Human capital. A theoretical and empirical analysis with special reference to education. Chicago, The University of Chicago Press.

Blau, P. y O. Duncan, (1967) The american occupational structure. Nueva York, The Free Press.

Bravo, D.; Sanhueza, C. y S. Urzúa, (2008) Is there labor market discrimination among professionals in Chile? Lawyers, doctors and business people. Documento de trabajo número 264. Santiago: Departamento de Economía, Universidad de Chile.

Brunner, J.J. y D. Uribe, (2007) Mercados universitarios: el nuevo escenario de la educación superior. Santiago, Ediciones Universidad Diego Portales.

Brunner, J.J., (2004) «Políticas y mercado de educación superior: necesidades de información». En Brunner, J.J. y Meller, P (Comp.) Oferta y demanda de profesionales y técnicos en Chile. El rol de la información pública. Santiago, Ril editores.

Bryk, A. y S. Raudenbush, (1992) Hierarchical lineal models: application and data analysis methods. Newbury Park, CA, Sage.

Budig, M., (2002) «Male advantage and the gender composition of jobs. Who tides the glass escalator?» en Social Problems. Número 49, (sin fecha), pp. 258-277.

Calvó - Armengol, A., (2006) «Redes sociales y mercado laboral» en Els Opuscles del Centre de Recerca en Economia Internacional (CREI). Universitat Pompeu Fabra. Número 17, Enero, 2006, 1 - 47.

Cohen, P. y M. Huffman, (2003) «Individuals, jobs, and labor markets: the devaluation of women's work» en American Sociological Review. Volumen 68. Número 3. Junio, 2003, 443 - 463. 
Contreras, D.; Gallegos, S. y F. Meneses, (2009) «Determinantes de desempeño universitario: iimporta la habilidad relativa? Análisis ex ante y ex post de una política pública» en Revista de Calidad en la Educación. Número 30, julio de 2009, pp. 18-31.

Elacqua, G., (2004) «Diagnóstico sobre los sistemas internacionales de información de la demanda de ocupaciones y la oferta de carreras» en Brunner, J.J. y P. Meller (comp.) Oferta y demanda de profesionales y técnicos en Chile: el rol de la información pública. Santiago, Ril editores.

Elfernan, R.; Soto, C.; Colbe, D. y J. Ramos, (2009) Determinantes de los salarios por carrera. Documento de trabajo número 300. Santiago, Departamento de Economía, Universidad de Chile.

Gallego, J.L., (2009) Apuntes de econometría. Santander, Departamento de Economía, Universidad de Cantabria.

Gallegos, S. y F. Meneses, (2007) « $i$ Es eficiente el sistema de ingreso a la universidad? El uso de ranking en la Pontificia Universidad Católica de Chile». Disponible en http://www.ideaseneducacion.cl/wp-content/ uploads/2008/07/paper-puc-sistema-de-ingreso3.pdf

Gibaja, J.J., (2008) Aspectos avanzados del modelo lineal generalizado. Bilbao, Orkestra-Instituto Vasco de Competitividad.

Henríquez, J., (2012) «Los silenciosos cambios que vienen para acortar carreras universitarias: nivelación, portabilidad y ciclos» en La Segunda, 6 de octubre de 2012.

Lara, B.; Meller, P. y G. Valdés, (2010) «Valor presente neto y tasa interna de retorno para diferentes carreras universitarias» en Meller, P. (Ed.) Carreras universitarias: rentabilidad, selectividad y discriminación. Santiago, Uqbar Editores.

Meller, P. y V. Quiroga, (2010a) «Evolución de la educación universitaria» en Meller, P. (Ed.) Carreras universitarias: rentabilidad, selectividad y discriminación. Santiago, Uqbar Editores.

Meller, P., (2010b) «Rol de la universidad en la determinación del ingreso de los profesionales» en Meller, P. (Ed.) Carreras universitarias: rentabilidad, selectividad y discriminación. Santiago, Uqbar Editores.

Meller, P., (2010c) «Perspectiva histórica de la discriminación femenina a nivel profesional» en Meller, P. (Ed.) Carreras universitarias: rentabilidad, selectividad y discriminación. Santiago, Uqbar Editores.

Mi Futuro, (2013) Base de datos de instituciones 2013. Buscador de Instituciones. Santiago, Ministerio de Educación. 
Mizala, A. y P. Romaguera, (2004) «Remuneraciones y tasas de retorno de los profesionales chilenos» en Brunner, J.J. y Meller, P. (Comp.) Oferta y demanda de profesionales y técnicos en Chile: el rol de la información pública. Santiago, Ril Editores.

Muñoz, A., (2012) «Rectores denuncian que Educación los obliga a acortar carreras y demora entrega de fondos» en El Mercurio, 28 de septiembre de 2012. C1

Neira, A.L. y D. Núñez, (2006) Requisitos formativos de ingreso a la administración pública. Estudios y documentos, Santiago, Consejo Nacional de Educación.

Núñez, J. y R. Gutiérrez, (2004a) Classism, discrimination and mericocracy in the labor market: the case of Chile. Documento de trabajo número 208. Santiago, Departamento de Economía, Universidad de Chile.

Núñez, J. y C. Risco, (2004b) Movilidad intergeneracional del ingreso en Chile. Santiago, Expansiva.

Núñez, J. y G. Pérez, (2007) Dime cómo te llamas y te diré quién eres: la ascendencia como mecanismo de diferenciación social en Chile. Documento de trabajo número 269, Santiago, Departamento de Economía, Universidad de Chile.

Organización para la Cooperación y el Desarrollo Económico (OCDE) / Banco Mundial (2009) La educación superior en Chile. Revisión de políticas nacionales de educación. Paris, Publicado por acuerdo con la OCDE, Paris y el BIRD/Banco Mundial.

Pardo, A.; Ruiz, M.A. y R. San Martín, (2007) «Cómo ajustar e interpretar modelos multinivel con SPSS» en Psicothema. Volumen 19. Número 2, 2007, pp. 308-321.

Rappoport, D.; Benavente, J.M. y P. Meller, (2004) Rankings de universidades chilenas según los ingresos de sus titulados. Documento de trabajo número 306. Santiago, Banco Central de Chile.

Rappoport, D.; Meller, P. y B. Lara, (2010) «Variabilidad de las remuneraciones de los profesionales» en Meller, P. (Ed.) Carreras universitarias: rentabilidad, selectividad y discriminación. Santiago, Uqbar Editores.

Requena, F., (1989) «El concepto de red social» en Revista Española de Investigaciones Sociológicas (REIS). Número 49. 1989, pp. 137 - 152.

Requena, F., (1991) Redes sociales y mercado de trabajo. Elementos para una teoría del capital relacional. Colección Monografías. Número 119. Madrid, Centro de Investigaciones Sociológicas. 
Rumberger, R. y S. Thomas, (1993) «The economic returns to college quality, major, and performance» en Economics of Education Review. Volumen 12. Número 1. Mayo, 1992, $1-19$.

Sapelli, C., (2009) Los retornos a la educación en Chile. Estimaciones por corte transversal y por cohortes. Documento de trabajo IE-PUC número 349. Santiago, Pontificia Universidad Católica.

Servicio de Información de la Educación Superior, (SIES), (2009a) Titulados de pregrado 2009 de Educación Superior. Disponible en: www.mifuturo.cl

Servicio de Información de la Educación Superior, (SIES), (2009b) Informe sobre duración real de las carreras y/o programas. Titulados 2009. Disponible en: www.mifuturo.cl Fecha de descarga: 31 de marzo de 2013.

Servicio de Información de la Educación Superior, (SIES), (2012a) Base de datos matriculados 2012. Disponible en: www.mifuturo.cl Fecha de descarga: 31 de marzo de 2013.

Servicio de Información de la Educación Superior, (SIES), (2012b) Compendio histórico. Disponible en: www.mifuturo.cl Fecha de descarga: 31 de marzo de 2013.

Urzúa, S., (2012) La rentabilidad de la educación superior en Chile. ¿Educación superior para todos? Documento de trabajo número 386. Santiago, Centro de Estudios Públicos (CEP).

Valdés, G.; Meller, P. y B. Lara, (2010) «Análisis de los diferenciales de remuneraciones entre los/as profesionales chilenos/as» en Meller, P. (Ed.) Carreras universitarias: rentabilidad, selectividad y discriminación. Santiago Uqbar Editores.

Zarhi, M., (2010) Educación superior y mercado del trabajo: factores que afectan la inserción laboral de jóvenes que son primera generación laboral con estudios terciarios. Tesis de Magíster en Sociología. Santiago, Pontificia Universidad Católica de Chile.

Recibido: 31/03/2013

Aceptado: 24/06/2013 